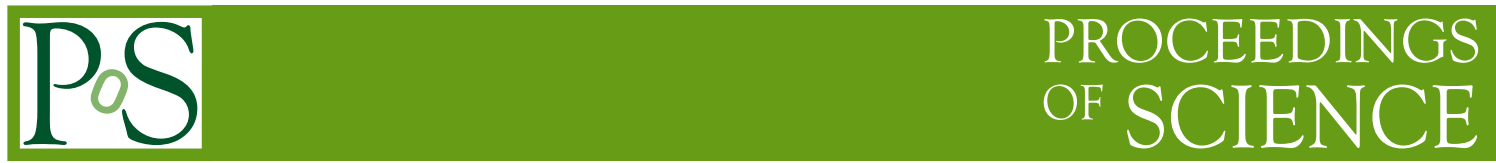

\title{
On the trace anomaly in $2+1$ flavor QCD
}

\section{P. Petreczky*t}

Physics Department, Brookhaven National Laboratory, Upton, NY 11973 USA

E-mail: petreczk@bnl.gov

We report on recent progress by the HotQCD collaboration in studying the trace anomaly at nonzero temperature in 2+1 flavor QCD on lattices with the temporal extent $N_{\tau}=4,6,8,10$ and 12 using the highly improved staggered quark (HISQ) action as well as the asqtad action. We discuss the dependence of our lattice results on the scale setting procedure and compare them with hadron resonance gas (HRG) calculations as well as to resummed perturbative results.

The 30 International Symposium on Lattice Field Theory - Lattice 2012,

June 24-29, 2012

Cairns, Australia

*Speaker.

† for HotQCD Collaboration: A. Bazavov, T. Bhattacharya, M. Buchoff, M. Cheng, N. Christ, C. DeTar, H.-T. Ding, S. Gottlieb, R. Gupta, P. Hegde, U. Heller, C. Jung, F. Karsch, E. Laermann, L. Levkova, Z. Lin, R. Mawhinney, S. Mukherjee, P. Petreczky, D. Renfrew, C. Schmidt, C. Schroeder, W. Soeldner, R. Soltz, R. Sugar, D. Toussaint, P. Vranas 


\section{Introduction}

At high temperatures strongly interacting matter undergoes an transition to a new state where quark and gluons are not subject to confinement; it shows up in a qualitative change in the equation of state (see [1] for a review). The most common way to calculate the QCD equation of state is the integral method. The pressure is calculated as an integral of the trace of the energy momentum tensor $\varepsilon-3 p$ or the trace anomaly

$$
\frac{p}{T^{4}}-\frac{p_{0}}{T_{0}^{4}}=\int_{T_{0}}^{T} d T^{\prime} \frac{\varepsilon-3 p}{T^{\prime 5}} .
$$

The study of the trace anomaly is also interesting in its own right. At high temperature it is determined by the running of the QCD coupling constant $\alpha_{s}$, and at leading order it is proportional $\alpha_{s}^{2} T^{4}$. Thus it provides a sensitive test of the weakly coupled nature of the quark gluon plasma. For this reason it is also often called the interaction measure. Thermodynamic quantities are expected to have large cutoff effects at high temperatures when calculated using an unimproved fermion formulation, even if the lattice spacing is small in absolute units. These cutoff effects arise from the distortion of the quark dispersion relation on the lattice and can be cured by using an improved action $[2,3]$. On the other hand, at low and intermediate temperatures the cutoff effects in thermodynamic quantities can be understood in terms of the cutoff effects of the hadron spectrum. In particular, the breaking of the so-called taste symmetry in the staggered fermion formulation could be the dominant source of cutoff effects when using staggered quarks. To control cutoff effects in pressure and other thermodynamic quantities, one first needs to understand the cutoff dependence of the trace anomaly. In this contribution we will study in detail the cutoff dependence of the trace anomaly and compare our lattice results with the hadron resonance gas model at low temperature and with resummed perturbation theory at high temperatures.
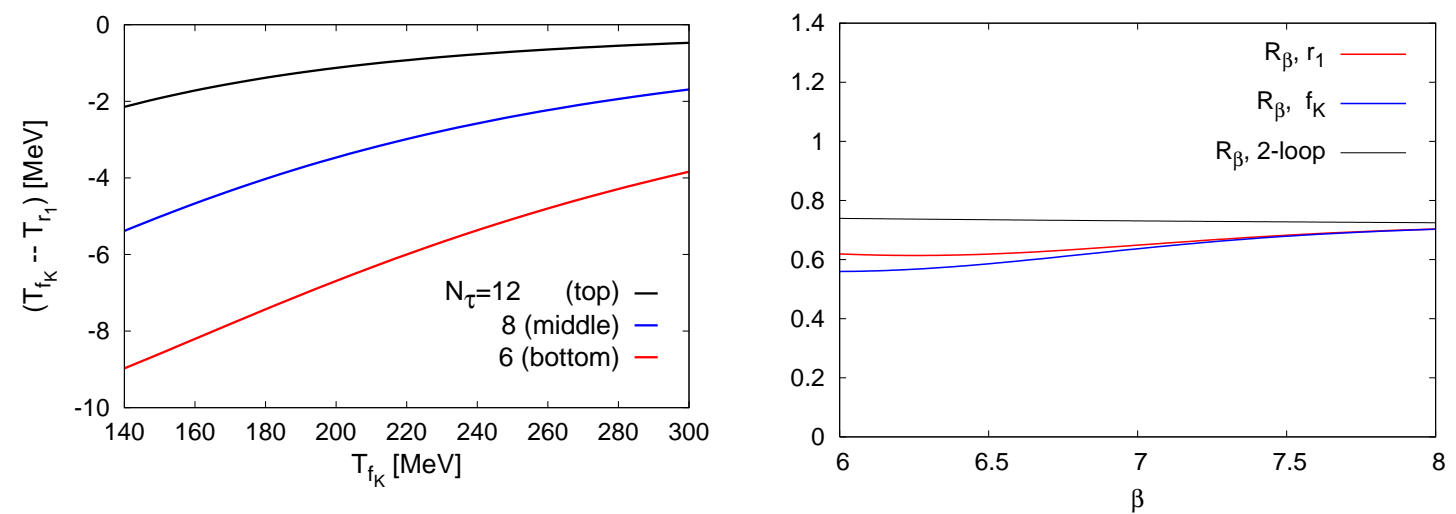

Figure 1: The difference in temperature (left) set by the $r_{1}$ and $f_{K}$ scales, described in the text. The running of the gauge coupling (right).

\section{Numerical results}

We calculate the interaction measure in $2+1$ flavor QCD using a tree-level improved action for gauge fields and the highly improved staggered quark (HISQ) action [4]. This combination 
of gauge and quark action is called the HISQ/tree action. It can largely reduce cutoff effects in thermodynamic quantities both at low and high temperatures $[5,6]$.

The trace anomaly can be written in terms of the expectation values of gauge action and quark condensates as

$$
\frac{\varepsilon-3 p}{T^{4}}=R_{\beta}\left[\left\langle S_{\text {gauge }}\right\rangle_{0}-\left\langle S_{\text {gauge }}\right\rangle_{T}\right]-R_{\beta} R_{m}\left[2 m_{l}\left(\langle\bar{l} l\rangle_{0}-\langle\bar{l} l\rangle_{T}\right)+m_{s}\left(\langle\bar{s} s\rangle_{0}-\langle\bar{s} s\rangle_{T}\right)\right],
$$

where the subscript " 0 " refers to $T=0$ and " $T$ " to finite temperature for observables evaluated at the same value of the cutoff. Furthermore, $R_{\beta}$ and $R_{m}$ are the lattice beta function and mass anomalous dimension that are defined below. The above expression is free of ultraviolet divergences. On a hypercubic lattice $N_{s}^{3} \times N_{\tau}$ the physical temperature is set by the size of the temporal dimension and the lattice spacing as $T=1 /\left(N_{\tau} a\right)$. For $T=0$ calculations we use $N_{\tau} \geqslant N_{s}$, and for $T>0$ we keep $N_{s} / N_{\tau}=4$ and at fixed $N_{\tau}$ vary the lattice spacing $a$ by varying the gauge coupling $\beta=10 / \mathrm{g}^{2}$. The continuum limit in this setup corresponds to $N_{\tau} \rightarrow \infty$. Therefore, we carried out this study on lattices with $N_{\tau}=4,6,8,10$ and 12 . The strange quark mass $m_{s}$ is tuned to the physical value, while the two degenerate light quarks have masses $m_{l}=m_{s} / 20$, slightly heavier than physical ( $m_{l} \simeq m_{s} / 27$ ). In the continuum limit these light quark masses correspond to the pion mass of about $160 \mathrm{MeV}$. To set the lattice spacing in physical units (fm) we use the $r_{1}$ scale defined through static quark potential

$$
\left.r^{2} \frac{d V}{d r}\right|_{r=r_{1}}=1 \text {. }
$$

We use the value $r_{1}=0.3106 \mathrm{fm}$ [7]. Alternatively, the kaon decay constant, $f_{K}=156.1 \mathrm{MeV}$ is used to set the scale. >From Fig. 1 (left) one can see how the choice of reference observables affects the conversion of temperature from lattice units to $\mathrm{MeV}$. Over the temperature range of interest on $N_{\tau}=6$ lattices the difference is within $9 \mathrm{MeV}$, and on $N_{\tau}=12$ within $2 \mathrm{MeV}$. Another effect of using different scales is related to the $\beta$-function and mass anomalous dimension that enter in Eq. $(2.1)$

$$
R_{\beta}(\beta)=-a \frac{d \beta}{d a}, R_{m}(\beta)=\frac{1}{m_{s}(\beta)} \frac{d m_{s}(\beta)}{d \beta},
$$

where $m_{s}(\beta)$ defines a line of constant physics (LCP), i.e., the combination of the gauge coupling and strange quark mass such that the kaon mass (in $\mathrm{MeV}$ ) stays approximately constant in the whole $\beta$ range used in the simulation. $R_{\beta}$ for $r_{1}$ and $f_{K}$ scale is shown in Fig. 1 (right), together with the 2-loop perturbative result.

The present status of the interaction measure with the HISQ/tree action is shown in Fig. 2 together with the stout continuum estimate of Ref [8]. For temperature $T<180 \mathrm{MeV}$ we see good agreement between our HISQ/tree results and the stout continuum estimate. At higher temperatures we see significant discrepancies between HISQ/tree results and the stout results, though the discrepancies seem to disappear at temperatures $T>350 \mathrm{MeV}$. The height of the peak decreases slightly when going from $N_{\tau}=8$ to $N_{\tau}=10$ and 12 . Thus one may expect that the discrepancies between HISQ/tree and stout actions in the peak region will be reduced once the continuum extrapolation for HISQ/tree is performed. It should be noted that in the previous calculations with $\mathrm{p} 4$ and asqtad actions $[9,10,11]$ the trace anomaly was significantly smaller due to large cutoff effects.

Next we study the effect of the scale setting on the trace anomaly. In Fig. 2 (right) the results for $N_{\tau}=6$ and 10 are shown using $r_{1}$ and $f_{K}$ to set the scale. Different scale settings affect the 

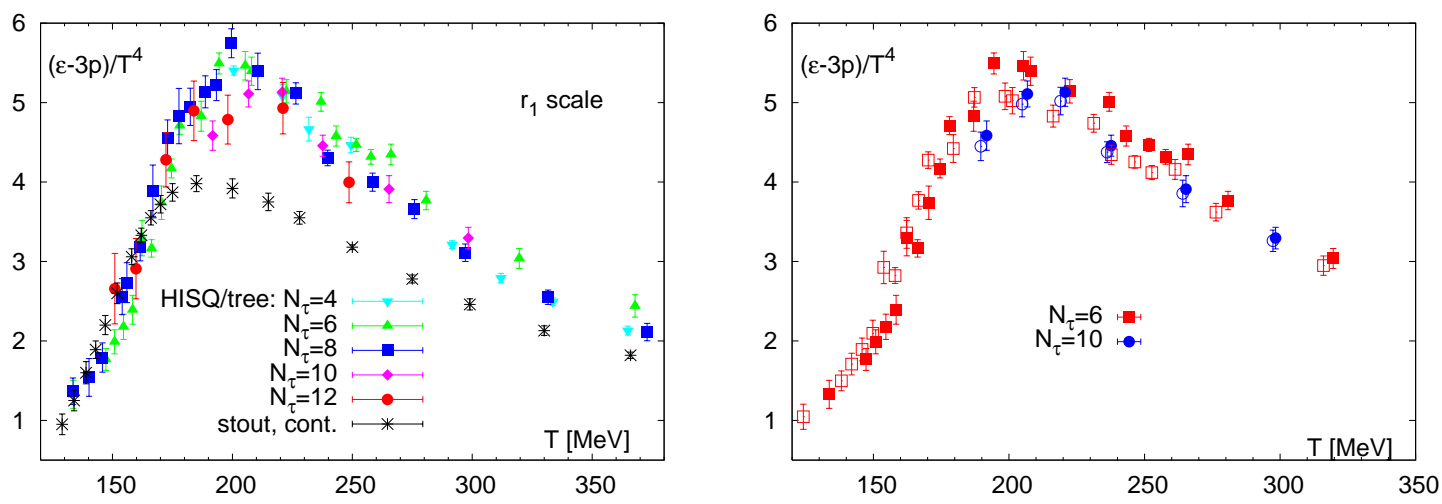

Figure 2: Comparison of the HISQ/tree interaction measure on $N_{\tau}=4,6,8,10$ and 12 lattices with the stout continuum estimate [8] (left), $N_{\tau}=6$ and $10 \mathrm{HISQ} /$ tree data (right). Filled (open) symbols in the right panel correspond to the $r_{1}\left(f_{K}\right)$ scale, see text.

$N_{\tau}=6$ results, in particular in the peak region the trace anomaly is smaller if $f_{K}$ is used to set the scale. However, for $N_{\tau}=10$ there is almost no difference between the two scale setting procedures.

We also calculated the trace anomaly using the asqtad action. The expression for the trace anomaly in terms of expectation values of local operators is given in Ref. [10]. In Fig. 3 we compare our results obtained with HISQ/tree action for $N_{\tau}=8$ and $N_{\tau}=12$ using the scale set with both $r_{1}$ (filled symbols) and $f_{K}$ (open symbols). If $r_{1}$ is used to set the scale, there is a significant discrepancy between the HISQ/tree and the asqtad result for $T<200 \mathrm{MeV}$ for $N_{\tau}=8$. As discussed above this is due to large cutoff effects at low temperatures when the asqtad action is used. The discrepancy is significantly smaller for $N_{\tau}=12$, as expected. If we use $f_{K}$ to set the scale, the discrepancies between HISQ/tree results and asqtad results are greatly reduced. Note, however, that the peak height in the $N_{\tau}=8$ asqtad data is reduced when $f_{K}$ is used to set the scale. Fig. 3 also shows that the difference between the two scale setting procedures for the asqtad action is reduced in the case of $N_{\tau}=12$ data. This is again expected. There is, however still a significant reduction in the peak height if $f_{K}$ scale is used.

The low-temperature behavior of the interaction measure is shown in Fig. 4 (left) and compared to the hadron resonance gas (HRG) calculation shown as the black line. At low temperatures, cutoff effects arising from the taste symmetry breaking of the staggered fermion formulation could be significant. To take into account possible cutoff effects in the comparison with HRG we replaced the contribution of each pion and kaon with a contribution averaged over the sixteen different tastes of pseudo-scalar mesons. The pseudo-scalar meson masses for each taste as function of the lattice spacing have been previously estimated by the HotQCD collaboration [6]. In Fig. 4 we show the HRG results with the modified pseudo-scalar meson sector as colored lines for each value of $N_{\tau}$. We use the same color coding for the lattice results and the modified HRG result. Despite the fact that even for HISQ/tree action the effects of taste breaking in the pseudo-scalar sector are significant, the resulting cutoff effects are surprisingly small. They are of the same order or smaller than the statistical errors for all $N_{\tau}$ values, including $N_{\tau}=6$. Fig. 4 shows that the lattice result starts to disagree with the HRG model at $T \sim 150-160 \mathrm{MeV}$.

Finally, let us compare our results for the trace anomaly with earlier calculations using the p4 action $[9,10]$ as well as with resummed perturbation theory [12]. In Fig. 4 (right) we compare 

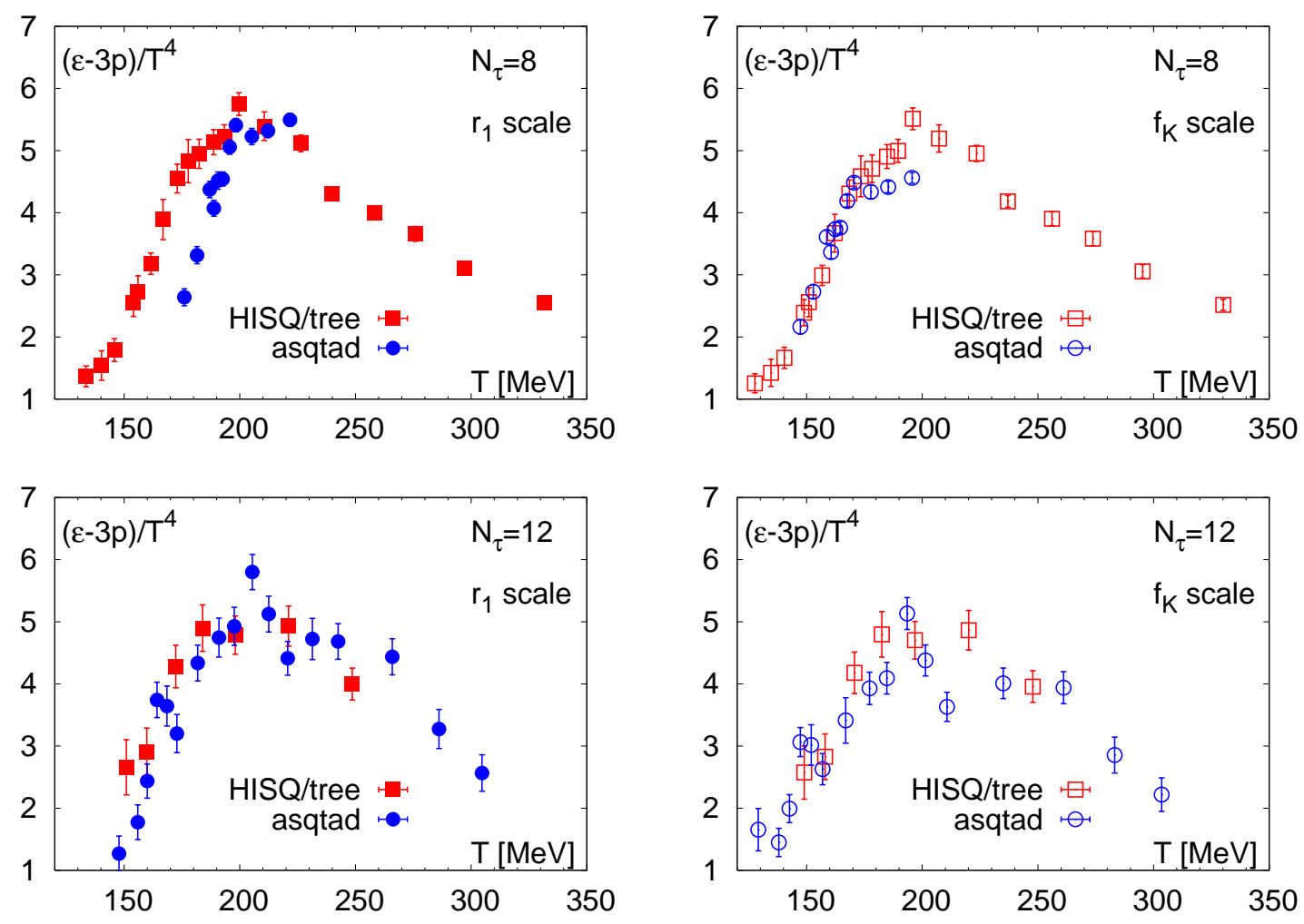

Figure 3: The interaction measure for the HISQ/tree action on $N_{\tau}=8$ (top) and $N_{\tau}=12$ (bottom) ensembles with $r_{1}$ (left) and $f_{K}$ (right) scale.

our results for the HISQ/tree action with previous results obtained with $\mathrm{p} 4$ action and the stout continuum estimate. For $T>400 \mathrm{MeV}$ different lattice data agree well with each other. The only exception is the $N_{\tau}=4 \mathrm{p} 4$ data, which show the expected cutoff effects at high temperatures. The $N_{\tau}=4 \mathrm{HISQ} /$ tree data should show similar cutoff dependence, but interestingly enough this is not observed. It is important to stress that at high temperatures the cutoff effects for p4 action should be small for $N_{\tau} \geq 6$. Thus the agreement between $\mathrm{p} 4$ results and HISQ/tree results is expected. We see quite good agreement with the resummed perturbative results [12].

\section{Conclusion}

We studied the trace anomaly in QCD using HISQ/tree and asqtad actions and lattices with temporal extent $N_{\tau}=4,6,8,10$ and 12. Using different observables to set the scale, $r_{1}$ and $f_{K}$, allows for a crude estimate of the magnitude of cutoff effects, which seem to be small at the finest $N_{\tau}=12$ lattices both for HISQ/tree and asqtad actions. Clearly to get reliable continuum results for the trace anomaly and thus for the equation of state the calculations on $N_{\tau}=10$ and 12 lattices need to be significantly extended. We also compared our lattice results with HRG at low temperatures and resummed perturbative results at high temperatures. The trace anomaly is well described by HRG for $T<150 \mathrm{MeV}$. We also find a good agreement between lattice and resummed perturbative results at high temperatures. 

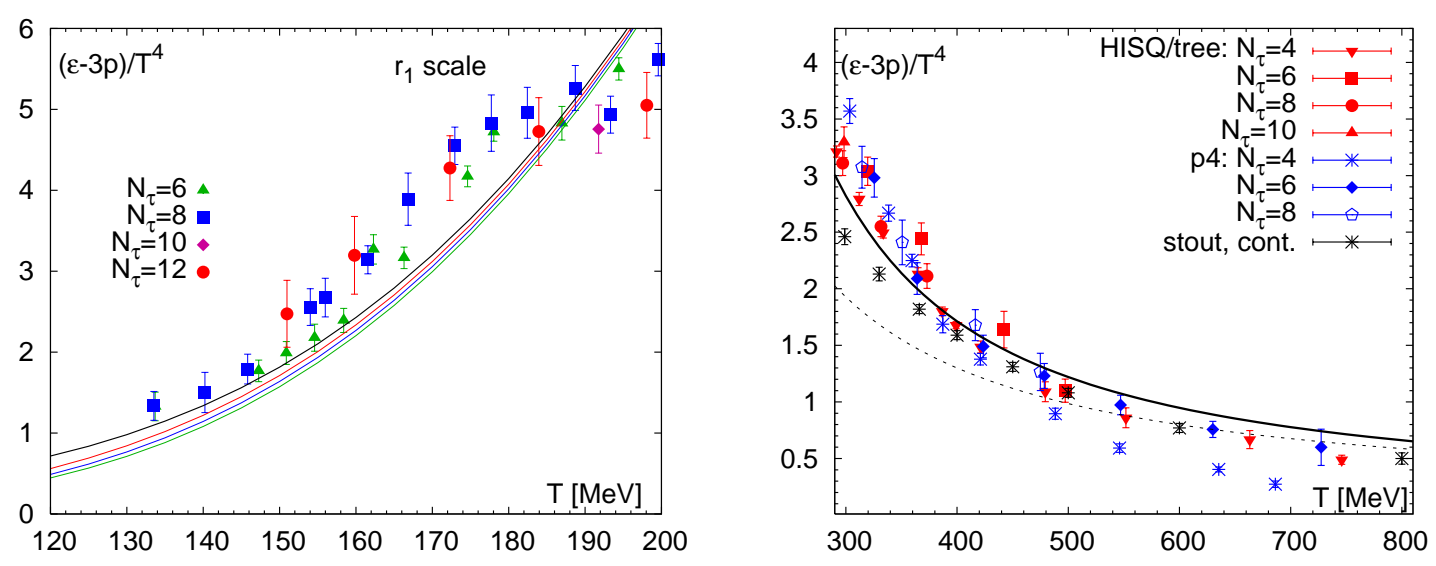

Figure 4: The interaction measure at low (left) and high (right) temperature. The curves on the left panel represent the HRG calculation, see text. On the right the dashed curve shows the resummed perturbative result with 2-loop running and the solid curve the resummed perturbative result with 1-loop running.

\section{Acknowledgments}

This work has been supported by contract DE-AC02-98CH10886 with the U.S. Department of Energy. The numerical simulations have been performed on BlueGene/L computers at Lawrence Livermore National Laboratory (LLNL), the New York Center for Computational Sciences (NYCCS) at Brookhaven National Laboratory, US Teragrid (Texas Advanced Computing Center), Cray XE6 at the National Energy Research Scientific Computing Center (NERSC), and on clusters of the USQCD collaboration in JLab and FNAL. The calculations for asqtad actions have been performed on BG/P computers of John von Neumann center in Jülich.

\section{References}

[1] P. Petreczky, J. Phys. G 39, 093002 (2012) [arXiv:1203.5320 [hep-lat]].

[2] U. M. Heller, F. Karsch and B. Sturm, Phys. Rev. D 60, 114502 (1999) [hep-lat/9901010].

[3] P. Hegde, F. Karsch, E. Laermann and S. Shcheredin, Eur. Phys. J. C 55, 423 (2008) [arXiv:0801.4883 [hep-lat]].

[4] E. Follana et al. (HPQCD/UKQCD Collaboration), Phys. Rev. D75, 054502 (2007), [arXiv:hep-lat/0610092].

[5] A. Bazavov et al. (HotQCD Collaboration), J. Phys. Conf. Ser. 230, 012014 (2010) [arXiv:1005.1131 [hep-lat]].

[6] A. Bazavov et al. (HotQCD Collaboration), Phys. Rev. D 85, 054503 (2012) [arXiv:1111.1710 [hep-lat]].

[7] A. Bazavov et al. (MILC Collaboration), Pos (Lattice 2010) 074 (2010) [arXiv:1012.0868 [hep-lat]].

[8] S. Borsanyi et al. (BW Collaboration), JHEP 1011:077 (2010) [arXiv:1007.2580 [hep-lat]].

[9] M. Cheng et al., Phys. Rev. D 77, 014511 (2008) [arXiv:0710.0354 [hep-lat]]. 
[10] A. Bazavov et al. (HotQCD Collaboration), Phys. Rev. D 80, 014504 (2009) [arXiv:0903.4379 [hep-lat]].

[11] M. Cheng et al., Phys. Rev. D 81, 054504 (2010) [arXiv:0911.2215 [hep-lat]].

[12] J. O. Andersen, L. E. Leganger, M. Strickland and N. Su, Phys. Rev. D 84, 087703 (2011) [arXiv:1106.0514 [hep-ph]]. 